\title{
Effect of deformation and orientation on spin orbit density dependent nuclear potential
}

\author{
Rajni Mittal ^, Raj Kumar, and Manoj K. Sharma \\ School of Physics and Materials Science, Thapar University, Patiala, Punjab, India.
}

\begin{abstract}
Role of deformation and orientation is investigated on spin-orbit density dependent part $\mathrm{V}_{J}$ of nuclear potential $\left(\mathrm{V}_{N}=\mathrm{V}_{P}+\mathrm{V}_{J}\right)$ obtained within semi-classical Thomas Fermi approach of Skyrme energy density formalism. Calculations are performed for ${ }^{24-54} \mathrm{Si}+{ }^{30} \mathrm{Si}$ reactions, with spherical target ${ }^{30} \mathrm{Si}$ and projectiles ${ }^{24-54} \mathrm{Si}$ having prolate and oblate shapes. The quadrupole deformation $\beta_{2}$ is varying within range of $0.023 \leq$ $\beta_{2} \leq 0.531$ for prolate and $-0.242 \leq \beta_{2} \leq-0.592$ for oblate projectiles. The spin-orbit dependent potential gets influenced significantly with inclusion of deformation and orientation effect. The spin-orbit barrier and position gets significantly influenced by both the sign and magnitude of $\beta_{2}$-deformation. Si-nuclei with $\beta_{2}<0$ have higher spin-orbit barrier (compact spin-orbit configuration) in comparison to systems with $\beta_{2}>0$. The possible role of spin-orbit potential on barrier characteristics such as barrier height, barrier curvature and on the fusion pocket is also probed. In reference to prolate and oblate systems, the angular dependence of spin-orbit potential is further studied on fusion cross-sections.
\end{abstract}

\section{Introduction}

The aspect of nuclear deformations has been continually probed to analyze the fusion processes. A lot of work has been done by taking one of the target or projectile as deformed nucleus [1-4]. When the nuclear deformations are incorporated in theoretical calculations, it is inherently necessary to account for the relative orientation of the projectile and the target in the fusion dynamics. The orientation of the colliding nuclei has a significant effect not only on the fusion barrier height but also on the compactness of the touching configuration. In order to have better understanding of fusion barrier, the contribution of nuclear interaction potential plays a significant role. In the Skyrme energy density formalism (SEDF) [5-7], the spinorbit dependent $\left(\mathrm{V}_{J}\right)$ and spin-orbit independent $\left(\mathrm{V}_{P}\right)$ potential add up to give the total nuclear potential. Therefore within this approach one can study effect of deformation and orientation on individual contribution of nuclear potential. In most of the previous studies [8,9], the spin-orbit part was overlooked by either studying nuclei with the major shell closed for both protons and neutrons or taking its contribution to be small. Evidently, the contribution of spin-orbit potential in fusion dynamics is appreciable [4].

In the present work, the role of deformation (quadrupole deformed $\beta_{2}$ ) and orientation is investigated on spin-orbit density dependent potential $\mathrm{V}_{J}$ of nuclear potential. Here, an effort is made to analyse the possible role of orientation degree of freedom on spin-orbit part via different $\beta_{2}$-deformed shapes. To facilitate discussion on $\mathrm{V}_{J}$ due to shape and orientation of deformed nuclei, calculations are performed for ${ }^{24-54} \mathrm{Si}+{ }^{30} \mathrm{Si}$ reactions having

\footnotetext{
^e-mail: rajni.mittal1989@gmail.com
}

quadrupole deformation $\left(\beta_{2}\right)$ within range of $0.023 \leq \beta_{2} \leq$ 0.531 (for prolate) and $-0.242 \leq \beta_{2} \leq-0.592$ (for oblate). Calculations are done within the SEDF using SKRA force [10]. The Skyrme parameter set obtained from SKRA force seem to address the nuclear interactions adequately.

The above mentioned reactions are chosen because the target ${ }^{30} \mathrm{Si}$ is spherical, whereas projectiles are weakly and highly deformed with prolate (p) and oblate (o) shapes. The ground state deformation of nuclei are taken from the work of Moller and Nix [11]. It is manifested from the calculations that, independent of the magnitude of prolate $\left(\beta_{2}>0\right)$ deformed nuclei, the spin-orbit barrier height $\left(\mathrm{V}_{J B}\right)$ (the spin-orbit barrier height $\left(\mathrm{V}_{J B}\right)$ is the maximum value of $\mathrm{V}_{J}$ and the corresponding position is spin-orbit barrier position $\left(\mathrm{R}_{J B}\right)$.) increases (corresponding spin orbit barrier position $\mathrm{R}_{J B}$ decreasing) from $\theta=0^{0}$ to $90^{\circ}$. For highly prolate nuclei, the spin-orbit barrier height and spin-orbit barrier position get influenced significantly in comparison to weakly prolate nuclei. In case of oblate shape nuclei $\left(\beta_{2}<0\right)$, irrespective of magnitude of deformation, the optimum angle (highest spin-orbit barrier and lower interaction radius) is obtained at $\left(0^{0}, 180^{\circ}\right)$. The effect of orientation on $\mathrm{V}_{J}$ is much prominent for nuclei with $\beta_{2}<0$ as compare to $\beta_{2}>0$. Further the effect of spin-orbit term is explored on fusion cross-sections $\left(\sigma_{\text {fusion }}\right)$ obtained by using Wong formula [12].

The purpose of this paper is two fold (i) to study the role of orientation on spin-orbit density part of nuclear potential. (ii) to see the effect of spin-orbit density interaction potential on fusion cross-section. In present study the role of quadrupole deformation is also studied via various prolate and oblate shape nuclei. 
This work is organized as follows: Section 2 contains the methodology, which includes Skyrme Energy Density Formalism (SEDF) and Wong formula. Calculations and results are discussed in Section 3 and finally the outcome is summarized in Section 4.

\section{Methodology}

\subsection{Semi-Classical Skyrme Energy Density Formalism (SEDF)}

The SEDF in semiclassical Extended Thomas Fermi (ETF) method [6] provides a convenient way for calculating the interaction potential between two nuclei. In the Hamiltonian density, the kinetic energy density $\tau$ as well as the spin-orbit density $\vec{J}$ are functions of the nucleon density $\rho_{q}, \mathrm{q}=\mathrm{n}, \mathrm{p}$. The nucleus-nucleus interaction potential in SEDF, based on semiclassical ETF model, is

$$
V_{N}(R)=E(R)-E(\infty)=\int H(\vec{r}) d \vec{r}-\int H_{1}(\vec{r}) d \vec{r}-\int H_{2}(\vec{r}) d \vec{r}
$$

Above equation which is nuclear interaction potential calculated by using slab approximation method as discussed later in reference to Eq. (3).

The Skyrme Hamiltonian density in Eq. (1) reads as,

$$
\begin{aligned}
H(\rho, \tau, \vec{J}) & =\frac{\hbar^{2}}{2 m} \tau \\
& +\frac{1}{2} t_{0}\left[\left(1+\frac{1}{2} x_{0}\right) \rho^{2}-\left(x_{0}+\frac{1}{2}\right)\left(\rho_{n}^{2}+\rho_{p}^{2}\right)\right] \\
& +\frac{1}{12} t_{3} \rho^{\alpha}\left[\left(1+\frac{1}{2} x_{3}\right) \rho^{2}-\left(x_{3}+\frac{1}{2}\right)\left(\rho_{n}^{2}+\rho_{p}^{2}\right)\right] \\
& +\frac{1}{4}\left[t_{1}\left(1+\frac{1}{2} x_{1}\right)+t_{2}\left(1+\frac{1}{2} x_{2}\right)\right] \rho \tau \\
& -\frac{1}{4}\left[t_{1}\left(x_{1}+\frac{1}{2}\right)-t_{2}\left(x_{2}+\frac{1}{2}\right)\right]\left(\rho_{n} \tau_{n}+\rho_{p} \tau_{p}\right) \\
& +\frac{1}{16}\left[3 t_{1}\left(1+\frac{1}{2} x_{1}\right)-t_{2}\left(1+\frac{1}{2} x_{2}\right)\right](\vec{\nabla} \rho)^{2} \\
& -\frac{1}{16}\left[3 t_{1}\left(x_{1}+\frac{1}{2}\right)+t_{2}\left(x_{2}+\frac{1}{2}\right)\right] \\
& \times\left[\left(\vec{\nabla} \rho_{n}\right)^{2}+\left(\vec{\nabla} \rho_{p}\right)^{2}\right] \\
& -\frac{1}{2} W_{0}\left[\rho \vec{\nabla} \cdot \vec{J}+\rho_{n} \vec{\nabla} \cdot \vec{J}_{n}+\rho_{p} \vec{\nabla} \cdot \vec{J}_{p}\right] \\
& -\frac{1}{16}\left[\left(t_{1} x_{1}+t_{2} x_{2}\right) \vec{J}^{2}+\left(t_{1}-t_{2}\right)\left(J_{p}^{2}+J_{n}^{2}\right)\right]
\end{aligned}
$$

Here, $\rho=\rho_{n}+\rho_{p}, \tau=\tau_{n}+\tau_{p}$, and $\vec{J}=\vec{J}_{n}+\vec{J}_{p}$ are the nuclear, kinetic energy and spin-orbit densities, respectively. $\mathrm{m}$ is the nucleon mass, and $x_{j}, t_{j}(j=0,1,2,3)$, $\alpha$ and $W_{0}$ are the Skyrme force parameters. In Eq. (2), the last two terms of Hamiltonian corresponds to spin-orbit effect. Author of [10] has introduced new Skyrme parameter set known as SKRA force, whose parameters are $\mathrm{t}_{0}=$ $-2895.4 \mathrm{MeV} \mathrm{fm}^{3}, \mathrm{t}_{1}=405.5 \mathrm{MeV} \mathrm{fm}^{5}, \mathrm{t}_{2}=-89.1 \mathrm{MeV}$ $\mathrm{fm}^{5}, \mathrm{t}_{3}=16660 \mathrm{MeV} \mathrm{fm}{ }^{3+3 \alpha}, \mathrm{x}_{0}=0.08, \mathrm{x}_{1}=0, \mathrm{x}_{2}=0.2$, $\mathrm{x}_{3}=0, \alpha=0.1422, \mathrm{~W}_{0}=129 \mathrm{MeV} \mathrm{fm}^{5}$. This force is found to account better for nuclear interactions and satisfactory describes finite nuclei at low and medium energy. Since our present study involves the nucleon interaction at low energy, such force is expected to give reasonably good results. For the proximity potential, Gupta et al. [13] introduce the slab approximation of semi-infinite nuclear matter with surfaces parallel to xy plane, moving in $\mathrm{z}$ direction, and separated by distance $s$ having minimum value $\mathrm{s}_{0}$. Then, the interaction potential $\mathrm{V}_{N}(\mathrm{R})$ between two nuclei separated by $R=R_{1}+R_{2}+s$ is given as (for more detail see Ref. [6, 13, 14]),

$$
\begin{aligned}
V_{N}(R)= & 2 \pi \bar{R} \int_{s_{0}}^{\infty} e(s) d s \\
= & 2 \pi \bar{R} \int\left\{H(\rho, \tau, \vec{J})-\left[H_{1}\left(\rho_{1}, \tau_{1}, \overrightarrow{J_{1}}\right)\right.\right. \\
& \left.\left.+H_{2}\left(\rho_{2}, \tau_{2}, \overrightarrow{J_{2}}\right)\right]\right\} d z \\
= & 2 \pi \bar{R} \int\left\{\left(H(\rho)-\left[H_{1}\left(\rho_{1}\right)+H_{2}\left(\rho_{2}\right)\right]\right)\right. \\
& \left.+\left(H(\vec{J})-\left[H_{1}\left(\vec{J}_{1}\right)+H_{2}\left(\vec{J}_{2}\right)\right]\right)\right\} d z \\
= & V_{P}(R)+V_{J}(R) .
\end{aligned}
$$

Here, $\mathrm{V}_{P}(\mathrm{R})$ and $\mathrm{V}_{J}(\mathrm{R})$ are spin-orbit density independent and dependent parts of nuclear interaction potential. $\bar{R}$ is the mean curvature radius defining the geometry of the system. Hence nuclear potential derived within the SEDF approach is a sum of spin-orbit dependent $\left(\mathrm{V}_{J}\right)$ and independent $\left(\mathrm{V}_{P}\right)$ part. Then, adding the Coulomb $\left(\mathrm{V}_{C}\right)$ and centrifugal $\left(\mathrm{V}_{\ell}\right)$ interactions to the nuclear interaction potential $\mathrm{V}_{N}(\mathrm{R})$, we get the total interaction potential for deformed and oriented nuclei [15], as

$$
\begin{aligned}
V_{T}(R)= & V_{N}\left(R, A_{i}, \beta_{\lambda i}, \theta_{i}, T\right) \\
& +V_{c}\left(R, Z_{i}, \beta_{\lambda i}, \theta_{i}, T\right) \\
& +V_{\ell}\left(R, A_{i}, \beta_{\lambda i}, \theta_{i}, T\right) .
\end{aligned}
$$

Here, $\beta_{\lambda i}, \lambda=2,3,4$ are the static quadrupole, octupole, and hexadecapole deformations and $i=1,2$ represents the projectile and target respectively. In the present work, the deformation effects up to quadrupole $\left(\beta_{2 i}\right)$ are included.

The deformation effect in Eq. (4) is introduced via the nuclear radii, as follows:

$$
R_{i}\left(\alpha_{i}, T\right)=R_{0 i}(T)\left[1+\sum_{\lambda} \beta_{\lambda i} Y_{\lambda}^{(0)}\left(\alpha_{i}\right)\right]
$$

Here, $\mathrm{R}_{0 i}$ is the half-density nuclear radius at temperature $\mathrm{T}=0$ obtained by fitting the experimental data to respective polynomials in the nuclear mass region $\mathrm{A}=4-238$ $[14,16]$. The T-dependence in the Eq. (5) is introduced as in [17]. In above expression, $\mathrm{T}$ is related to the incoming center-of-mass energy $\mathrm{E}_{c . m}$. or the compound nucleus $(\mathrm{CN})$ excitation energy $\mathrm{E}_{C N}^{*}$ through semi-classical statistical relation [18] as

$$
E_{C N}^{*}=\frac{A_{C N}}{8} T^{2}-T
$$



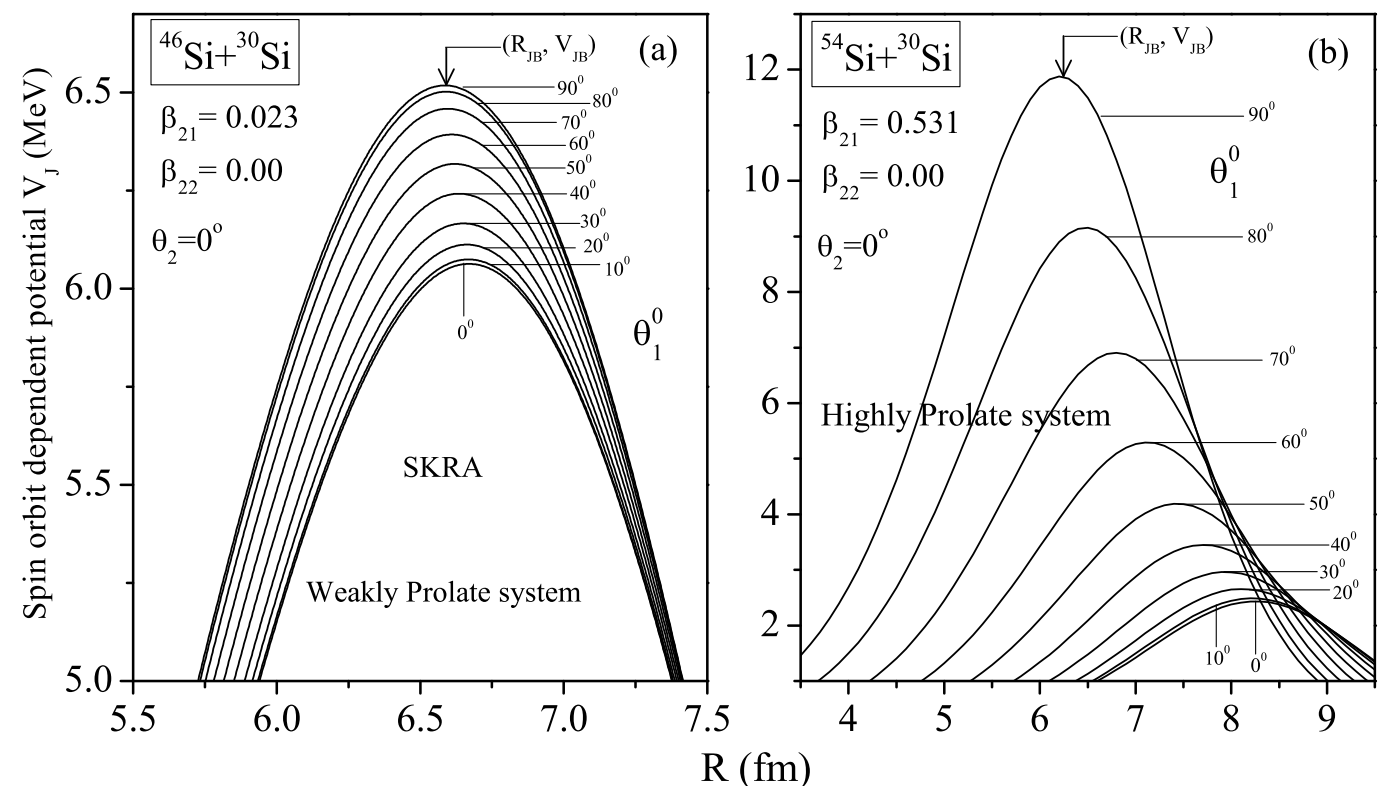

Figure 1. Figure shows the variation of spin-orbit dependent part $\mathrm{V}_{J}(\mathrm{R})$ of interaction potential, plotted for different orientation degree of freedom $\theta$ (a) for weakly prolate and (b) for highly prolate deformed nuclei. $\mathrm{V}_{J B}$ represent the maximum value of $\mathrm{V}_{J}$ and $\mathrm{R}_{J B}$ is corresponding position, different for different orientations.

\subsection{Wong formula}

According to Wong [12], the fusion cross-section, in terms of angular-momentum $\ell$ partial waves, for deformed and oriented nuclei (with orientation angles $\theta_{i}$ ), lying in same planes, and colliding with center-of-mass energy $\left(E_{c . m .}\right)$, is

$$
\sigma\left(E_{c . m .}, \theta_{i}\right)=\frac{\pi}{k^{2}} \sum_{\ell}(2 \ell+1) P_{\ell}\left(E_{c . m .}, \theta_{i}\right)
$$

with $k=\sqrt{\frac{2 \mu E_{c . m .}}{\hbar^{2}}}$, and $\mu$ as the reduced mass. Here, the penetrability $P_{\ell}$ is describes by the Hill-Wheeler $[19,20]$ approximation,

$$
P_{\ell}=\left[1+\exp \left(\frac{2 \pi\left(V_{B}^{\ell}\left(E_{c . m .}, \theta_{i}\right)-E_{c . m .}\right)}{\hbar \omega_{\ell}\left(E_{c . m .}, \theta_{i}\right)}\right)\right]^{-1},
$$

with $\hbar \omega_{\ell}\left(E_{c . m .}, \theta_{i}\right)$, evaluated at the barrier position $R=R_{B}^{\ell}$ corresponding to the maximum barrier height $V_{B}^{\ell}\left(E_{c . m .}, \theta_{i}\right)$.

Wong has applied some approximations, and replaced the $\ell$-summation in Eq.(7) by an integral, giving the fusion cross-section [12] as,

$$
\sigma\left(E_{c . m .}, \theta_{i}\right)=\frac{R_{B}^{0^{2}} \hbar \omega_{0}}{2 E_{c . m .}} \ln \left[1+\exp \left(\frac{2 \pi}{\hbar \omega_{0}}\left(E_{c . m .}-V_{B}^{0}\right)\right)\right],
$$

Here, one may use the interaction between deformed nuclei of radii $R_{i}$ with deformation parameters $\beta_{\lambda i}$ and making orientation angles $\theta_{i}$ with respect to the collision axis. Hence, the fusion cross-section integrated over the angles $\theta_{i}(\mathrm{i}=1,2)$ is obtained as

$$
\sigma\left(E_{c . m .}\right)=\int_{\theta_{1}, \theta_{2}=0}^{\pi / 2} \sigma\left(E_{c . m .}, \theta_{1}, \theta_{2}\right) \sin \theta_{1} d \theta_{1} \sin \theta_{2} d \theta_{2} \text {. }
$$

\section{Calculation and Result}

Within the framework of Skyrme Energy Density Formalism (SEDF), the role of deformation and orientation of colliding nuclei is investigated on spin-orbit density part $\mathrm{V}_{J}$ of nuclear potential $\left(\mathrm{V}_{N}=\mathrm{V}_{P}+\mathrm{V}_{J}\right)$ using SKRA force [10]. For the purpose of study, various $\mathrm{Si}$-induced reactions i.e. ${ }^{24} \mathrm{Si}+{ }^{30} \mathrm{Si},{ }^{26} \mathrm{Si}+{ }^{30} \mathrm{Si},{ }^{28} \mathrm{Si}+{ }^{30} \mathrm{Si},{ }^{38} \mathrm{Si}+{ }^{30} \mathrm{Si}{ }^{40} \mathrm{Si}+{ }^{30} \mathrm{Si}$, ${ }^{42} \mathrm{Si}+{ }^{30} \mathrm{Si},{ }^{44} \mathrm{Si}+{ }^{30} \mathrm{Si},{ }^{46} \mathrm{Si}+{ }^{30} \mathrm{Si},{ }^{48} \mathrm{Si}+{ }^{30} \mathrm{Si}, \quad{ }^{50} \mathrm{Si}+{ }^{30} \mathrm{Si}$, ${ }^{52} \mathrm{Si}+{ }^{30} \mathrm{Si}$ and ${ }^{54} \mathrm{Si}+{ }^{30} \mathrm{Si}$ are considered. Above mentioned reactions are so chosen because, the target is spherical and projectiles corresponds to either prolate or oblate shapes. It is to be mentioned here that, for the nuclei which are not discovered experimentally, the relevant details are taken from the theoretical work of Moller and Nix [11].

First of all, we study the effect of orientation on spinorbit potential by considering weakly and highly prolate systems i.e. ${ }^{46} \mathrm{Si}+{ }^{30} \mathrm{Si}$ and ${ }^{54} \mathrm{Si}+{ }^{30} \mathrm{Si}$ respectively as shown in left and right panels of figure 1 . The variation of $\mathrm{V}_{J}$ is shown for different orientation degree of freedom $\theta$ i.e. from $0^{0}$ to $90^{\circ}$. It is observed in figure 1 (a) that, for weakly prolate system with $\beta_{21}=0.023$ and $\beta_{22}=0.00, \mathrm{~V}_{J}$ is increasing slowly with the change in orientation of projectile from $0^{0}$ to $90^{\circ}$. At an orientation $\theta=0^{0}$, the height of spin-orbit barrier is $\sim 6.0 \mathrm{MeV}$, which for orientation angle of $\theta=90^{\circ}$, increases to $\sim 6.5 \mathrm{MeV}$. Apparently, the spinorbit barrier position $\mathrm{R}_{J B}$ is also affected by the orientation angles between the projectile and target as the maximum $\mathrm{R}_{J B}$ corresponds to $\theta_{1}=0^{0}$ and the minimum to $\theta_{1}=90^{\circ}$. On changing the orientation from $\theta_{1}=0^{0}$ to $90^{\circ}$, the spin-orbit barrier position is shifting $\sim 0.1 \mathrm{fm}$ towards lower interaction radius, making system more compact. 

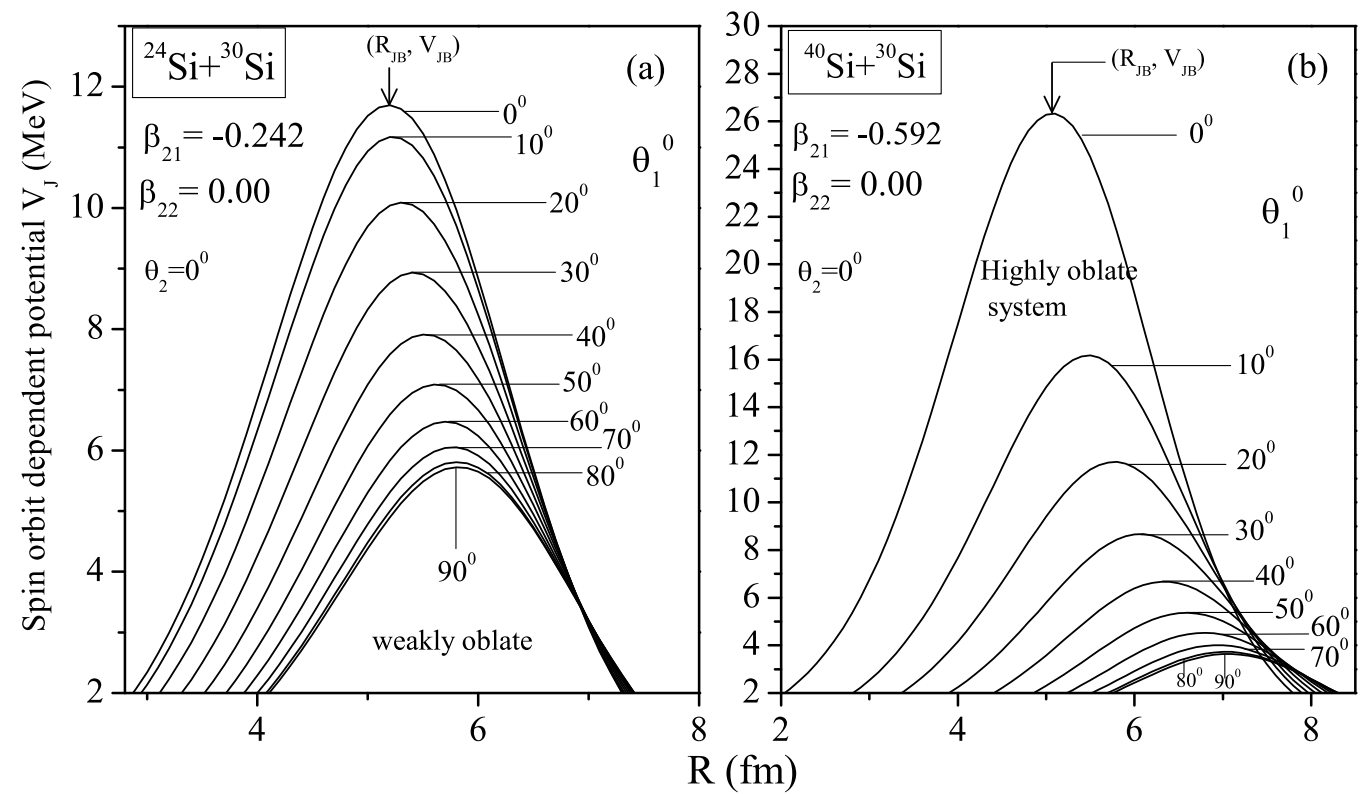

Figure 2. Same as Figure 1 but for oblate shape nuclei.

Table 1. The spin-orbit barrier height $\mathrm{V}_{J B}$ and spin-orbit barrier position $\mathrm{R}_{J B}$ of various prolate and oblate deformed systems with their optimum orientations [21]. The coulomb barrier $\mathrm{V}_{C B}$ is also shown in table below. $\beta_{2}$-deformations are taken from the theoretical work of Moller and Nix [11]. $\beta_{21}$ and $\beta_{22}$ represents the deformation of projectile and target respectively.

\begin{tabular}{lcccccccc}
\hline $\begin{array}{l}\mathrm{A}_{P} \\
(\mathrm{Si})\end{array}$ & $\begin{array}{l}\mathrm{A}_{T} \\
(\mathrm{Si})\end{array}$ & $\theta_{1}$ & $\theta_{2}$ & $\beta_{21}$ & $\beta_{22}$ & $\begin{array}{l}\mathrm{V}_{C B} \\
(\mathrm{MeV})\end{array}$ & $\begin{array}{l}\mathrm{V}_{J B} \\
(\mathrm{MeV})\end{array}$ & $\begin{array}{l}\mathrm{R}_{J B} \\
(\mathrm{fm})\end{array}$ \\
\hline 38 & 30 & 90 & 0 & 0.234 & 0 & 26.360 & 8.185 & 6.0 \\
46 & 30 & 90 & 0 & 0.023 & 0 & 25.092 & 6.526 & 6.6 \\
52 & 30 & 90 & 0 & 0.490 & 0 & 25.254 & 11.323 & 6.2 \\
54 & 30 & 90 & 0 & 0.531 & 0 & 25.119 & 11.875 & 6.2 \\
\hline \multicolumn{8}{c}{ Oblate deformed nuclei } \\
\hline 24 & 30 & 180 & 0 & -0.242 & 0 & 29.320 & 11.695 & 5.2 \\
26 & 30 & 180 & 0 & -0.353 & 0 & 29.266 & 14.548 & 5.1 \\
28 & 30 & 180 & 0 & -0.478 & 0 & 29.351 & 19.11 & 4.9 \\
40 & 30 & 180 & 0 & -0.592 & 0 & 29.975 & 26.321 & 5.1 \\
42 & 30 & 180 & 0 & -0.321 & 0 & 26.554 & 13.169 & 5.7 \\
44 & 30 & 180 & 0 & -0.263 & 0 & 26.110 & 11.502 & 5.9 \\
48 & 30 & 180 & 0 & -0.398 & 0 & 26.232 & 16.467 & 5.7 \\
50 & 30 & 180 & 0 & -0.422 & 0 & 26.154 & 17.794 & 5.7 \\
\hline
\end{tabular}

Similar results are obtained for highly prolate system i.e. for ${ }^{54} \mathrm{Si}+{ }^{30} \mathrm{Si}$ reaction with $\beta_{21}=0.531$ and $\beta_{22}=0.00$ as depicted in figure $1(\mathrm{~b})$. One may clearly see that, the enhancement in magnitude of $\mathrm{V}_{J}$ with respect to orientation degree of freedom is significantly high for highly prolate as compare to weakly prolate system. At an orientation angle of $90^{\circ}, \mathrm{V}_{J}$ of highly prolate system is $\sim 6$ $\mathrm{MeV}$ higher than weakly prolate system.

Further, the effect of orientation on spin-orbit part is analyzed through weakly and highly oblate shape nuclei as shown in figure 2. It is evident from the figure that, regardless of magnitude of deformation, the decrement in magni- tude of spin-orbit barrier height $\mathrm{V}_{J B}$ is observed from orientation $0^{0}$ to $90^{\circ}$ (see figure 2(a) and figure 2(b)). The higher spin-orbit barrier and lowest spin-orbit position is attained at $\theta_{1}=0^{0}, \theta_{2}=180^{\circ}$. On changing the orientation of projectile from $0^{0}$ to $90^{\circ}$, the $\mathrm{V}_{J}$ get affected as following: (i) the $\mathrm{V}_{J B}$ of weakly oblate system is decreased by a factor of $\sim 6.0 \mathrm{MeV}$ and shifted the spin-orbit barrier position $\sim 0.6 \mathrm{fm}$ towards higher interaction radius i.e. more elongated. (ii) for highly oblate system, the $\mathrm{V}_{J B}$ is decreased by $\sim 23.0 \mathrm{MeV}$ and $\mathrm{R}_{J B}$ moved $\sim 2.0 \mathrm{fm}$ towards higher interaction radius. 


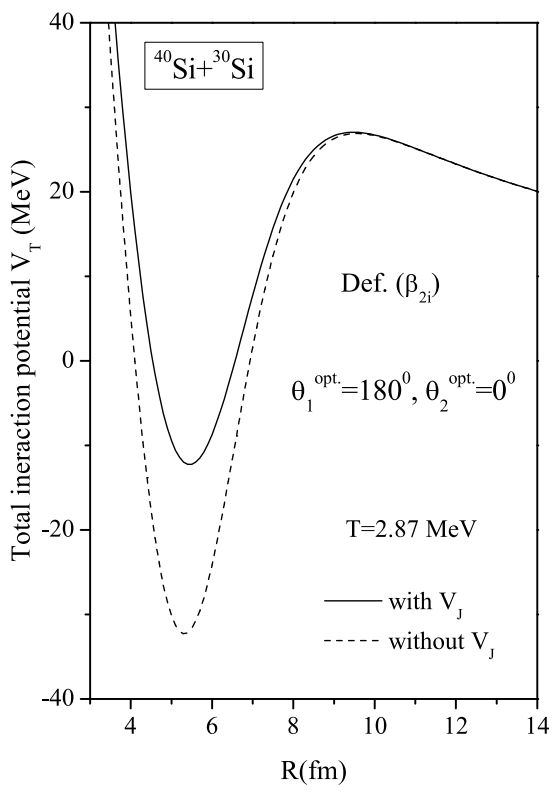

Figure 3. Total interaction potential $\mathrm{V}_{T}(\mathrm{MeV})$ of ${ }^{40} \mathrm{Si}+{ }^{30} \mathrm{Si}$ reaction as a function of inter nuclear distance $\mathrm{R}(\mathrm{fm})$ plotted with and without spin-orbit density effects at optimum orientations $\theta_{1}^{\text {opt. }}=180^{\circ}$ and $\theta_{2}^{\text {opt. }}=0^{0}$.

Moreover, on comparing $\mathrm{V}_{J B}$ of weakly prolate (figure 1(a)) and weakly oblate system (figure 2(a)) at their respective optimum orientations, we find that, in comparison to weakly prolate system, $\mathrm{V}_{J B}$ of weakly oblate system is increased $\sim 5.2 \mathrm{MeV}$. However, $\mathrm{V}_{J B}$ of highly oblate system is $\sim 14.4 \mathrm{MeV}$ higher than highly prolate system (see figure 1(b) and 2(b)). This analysis is further extended for other $\mathrm{Si}+\mathrm{Si}$ reactions, where magnitude of deformation is in between $0.023 \leq \beta_{2} \leq 0.531$ for prolate and $-0.242 \leq$ $\beta_{2} \leq-0.592$ for oblate systems. All prolate/oblate projectiles show similar result as we discussed above (therefore not shown here to avoid repetition). Table 1 summarizes the result for all considered reactions with their spin-orbit barrier height and position at the optimum orientations $\left(\theta_{1}\right.$, $\left.\theta_{2}\right)$.

The present study suggest that, the magnitude of prolate/oblate deformations and corresponding orientation angle between the colliding nuclei, significantly influence the spin-orbit barrier height and position. Moreover, the compactness of the system also get affected within the sign of $\beta_{2}$-deformation.

Now in order to see the role of spin-orbit part in the total interaction potential $\left(\mathrm{V}_{T}=\mathrm{V}_{P}+\mathrm{V}_{J}+\mathrm{V}_{C}=\mathrm{V}_{N}+\mathrm{V}_{C}\right)$, the spin-orbit independent potential $\left(\mathrm{V}_{P}\right)$ and Coulomb potential $\left(\mathrm{V}_{C}\right)$ parts are added to it. figure 3 shows the total interaction potential with and without the contribution of spin-orbit effect for ${ }^{40} \mathrm{Si}+{ }^{30} \mathrm{Si}$ reaction plotted at Coulomb barrier energy i.e. $\mathrm{E}_{C B}=29.98 \mathrm{MeV}$ (temperature $\mathrm{T}=2.87 \mathrm{MeV}$ ). It is clear that, impact of spin-orbit part is significant on fusion pocket rather than on fusion barrier height. Pocket becomes more shallower with inclusion of $\mathrm{V}_{J}$. Moreover, the barrier curvature $(\hbar \omega)$ also

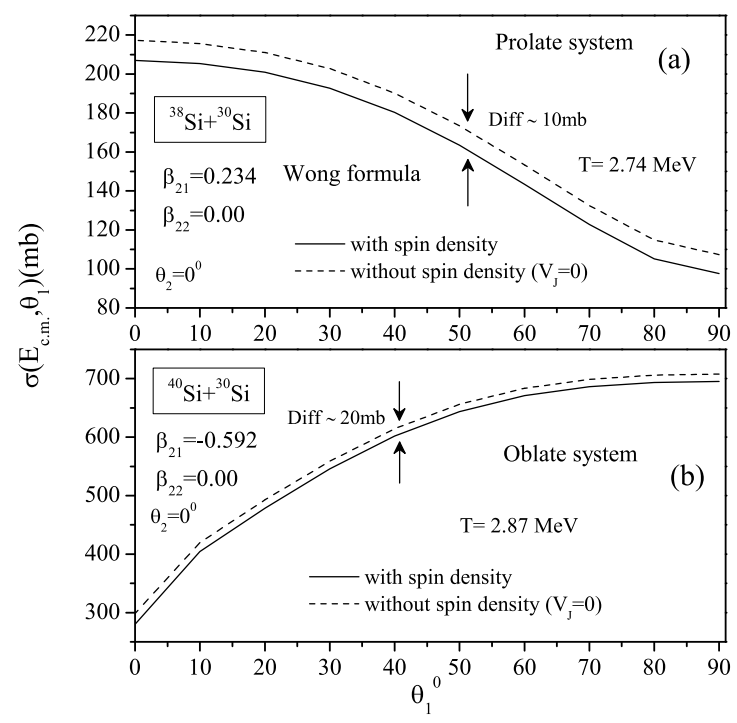

Figure 4. Variation of fusion cross-section $\sigma_{\text {fusion }}$ at each angle with and without spin density effects (a) for prolate and (b) for oblate shape nuclei, plotted at their coulomb barrier energy (see Table 1).

gets reduced with the inclusion of spin-orbit potential. In view of this point, it would be of further interest to study the influence of spin-orbit part on fusion cross-sections. The fusion cross-sections are calculated with and without the inclusion of spin-orbit density part in the total interaction potential and plotted at different orientations as shown in figure 4. Calculations are performed for ${ }^{38} \mathrm{Si}+{ }^{30} \mathrm{Si}$ and ${ }^{40} \mathrm{Si}+{ }^{30} \mathrm{Si}$ reactions with respective prolate and oblate shapes. Since no experimental data is available for these reactions, therefore cross-sections are estimated at their respective Coulomb barrier energy (see Table 1). It is observed that, the spin-orbit density part decreases the fusion cross-sections to a small extent within the Wong formula. This fall off in cross-section is about $\sim 10 \mathrm{mb}$ for prolate system and $\sim 20 \mathrm{mb}$ for oblate system. As expected, for prolate system (as shown in figure 4(a)), independent of spin orbit effect, the fusion cross-sections are decreasing with increase in orientation angle of colliding nuclei with maxima at $\theta_{2}=0^{0}$. On the other hand, for oblate nuclei, $\sigma_{\text {fusion }}$ increases with increase in orientation angle of colliding nuclei and become maximum at $\theta_{2}=90^{\circ}$ as shown in figure 4(b). For in between configurations the fusion cross-sections vary smoothly as a function of orientation angle.

\section{Summary}

A systematic study is carried out to see the influence of deformation and orientation on spin-orbit density part $\mathrm{V}_{J}$ by employing various prolate and oblate shape nuclei within the Skyrme Energy Density Formalism. The deformation is included upto $\beta_{2}$ lying within range of $0.023 \leq \beta_{2} \leq$ 0.531 for prolate and $-0.242 \leq \beta_{2} \leq-0.592$ for oblate nuclei. We observed that, independent of magnitude of pro- 
late deformed nuclei, higher spin-orbit barrier and lowest spin-orbit barrier position corresponds to orientation $90^{\circ}$, which for oblate shape nuclei changes to orientation angle $0^{0}$. The spin-orbit barrier and position is significantly influenced by both the sign and magnitude of deformation. The oblate shape nuclei $\left(\beta_{2}<0\right)$ exhibit higher spinorbit barrier (and lowest barrier position) in comparison to prolate systems $\left(\beta_{2}>0\right)$. Also, the pocket of total interaction potential become more shallower with the inclusion of spin-orbit effect. In addition to this, the angular dependence of spin-orbit part is studied on fusion cross-section by taking both prolate and oblate systems. It is observed that, fusion cross-sections are maximum at $\theta_{2}=0^{0}$ for prolate and $\theta_{2}=90^{\circ}$ for oblate nuclei and their magnitude reduces slightly after inclusion of spin-density effect.

\section{Acknowledgment}

The financial support from the department of science and technology (DST), is gratefully acknowledged.

\section{References}

[1] A. Iwamoto and P. Moller, Nucl. Phys. A 605, 334 (1996).

[2] D. Jain, R. Kumar and M. K. Sharma, Nucl. Phys. A 915, 106-124 (2013).

[3] Rajni, R. Kumar and M. K. Sharma, Phys. Rev. C 90, 044604 (2014).

[4] D. Jain, M. K. Sharma, Rajni, R. Kumar and R. K. Gupta, Eur. Phys. J. A 50, 155 (2014).

[5] D. Vautherin and D. M. Brink, Phys. Rev. C 5, 626 (1972).
[6] R. K. Gupta, D. Singh, and W. Greiner, Phys. Rev C 75, 024603 (2007).

[7] J. Bartel and K. Bencheikh, Eur. Phys. J. A 14, 179 (2002).

[8] C. Ngo et al., Nucl. Phys. A 240, 353 (1975); C. Ngo et al., Nucl. Phys. A 252, 237 (1975).

[9] Fl. Stancu and D. M. Brink, Nucl. Phys. A 270, 236 (1976).

[10] M. Rashdan, Mod. Phys. Lett. 15, 1287 (2000).

[11] P. Moller and J. R. Nix, Nucl. Phys. A 361, 117 (1981).

[12] C. Y. Wong, Phys. Rev. Lett. 31, 766 (1973).

[13] P. Chattopadhyay and R. K. Gupta Phys. Rev. C 30, 1191 (1984).

[14] R. K. Gupta, D. Singh, R. Kumar, and W. Greiner, J. Phys. G: Nucl. Part. Phys. 36, 075104 (2009).

[15] R. K. Gupta, M. Balasubramaniam, R. Kumar, N. Singh, M. Manhas, and W. Greiner, J. Phys. G: Nucl. Part. Phys. 31, 631 (2005).

[16] L. R. B. Elton, Nuclear Sizes (Oxford University Press, London, 1961); H. de Vries, C. W. de Jager, and C. de Vries, At. Data Nucl. Data Tables 36, 495 (1987).

[17] S. Shlomo and J. B. Natowitz, Phys. Rev. C 44, 287 (1991).

[18] K. J. LeCouteur and D. W. Lang, Nucl. Phys. 13, 32 (1959).

[19] D. L. Hill and J. A. Wheeler, Phys. Rev. 89, 1102 (1953).

[20] T. D. Thomas, Phys. Rev. 116, 703 (1959).

[21] R. K. Gupta, M. Balasubramanium, R. Kumar, N. Singh, M. Manhas, and W. Greiner, J. Phys. G: Nucl. Part. Phys. 31, 631 (2005). 